\title{
Ultrafast metal-semiconductor-metal photodetectors on low-temperature-grown GaN
}

\author{
M. Mikulics, ${ }^{\text {a) }}$ M. Marso, P. Javorka, P. Kordoš, and H. Lüth \\ Institute of Thin Films and Interfaces and cni, Center of Nanoelectronic Systems for Information \\ Technology, Research Centre Jülich, D-52425 Jülich, Germany \\ M. Kočan and A. Rizzi \\ IV. Physikalisches Institut, Georg-August Universität Göttingen, D-37077 Göttingen, Germany \\ S. Wu and R. Sobolewski ${ }^{\text {b) }}$ \\ Department of Electrical and Computer Engineering and the Laboratory for Laser Energetics, \\ University of Rochester, Rochester New York 14627-0231
}

(Received 10 December 2004; accepted 18 April 2005; published online 17 May 2005)

\begin{abstract}
We have fabricated and characterized ultrafast metal-semiconductor-metal photodetectors based on low-temperature-grown (LT) GaN. The photodetector devices exhibit up to $200 \mathrm{kV} / \mathrm{cm}$ electric breakdown fields and subpicosecond carrier lifetime. We recorded as short as 1.4-ps-wide electrical transients using 360-nm-wavelength and 100-fs-duration laser pulses, that is corresponding to the carrier lifetime of $720 \mathrm{fs}$ in our LT GaN material. (C) 2005 American Institute of Physics.
\end{abstract} [DOI: $10.1063 / 1.1938004]$

Gallium nitride is a wide, direct band-gap semiconductor that makes it a very promising candidate for the fabrication of ultraviolet (UV) photodetectors. Various types of $\mathrm{GaN}$ photodetectors have been demonstrated recently, such as the Schottky barrier detector, ${ }^{1} p-n$ junction, ${ }^{2} p$-i-n structure, ${ }^{3}$ and metal-semiconductor-metal (MSM) photodiode. ${ }^{4}$ Among these, MSM photodetectors exhibit superior performance in terms of the response speed, device noise, and fabrication simplicity. However, the GaN photodetector performance varied from sample to sample depending on the material quality, ${ }^{5-8}$ in which growth temperature plays a major role. High quality GaN photodetectors with full width at half maximum (FWHM) of the response in the picosecond range $(\sim 3.5 \mathrm{ps})$, which are the best published data, were presented by Li et al. ${ }^{9,10}$ In this letter, we present the fabrication and properties of MSM photodetectors based on lowtemperature-grown (LT) GaN. Such material exhibits lower electron mobility and higher concentration of defects, thus subpicosecond carrier lifetime can be assumed, which essentially shortens the response time of these LT GaN photodetectors.

The photodetector structure was grown by plasma induced molecular beam epitaxy. An AlN/GaN/AlN/GaN heterostructure buffer with thickness of 100/1500/5/500 nm was deposited on $6 \mathrm{H} i$-SiC substrate. Subsequently, the active $\mathrm{GaN}$ layer was grown at $650{ }^{\circ} \mathrm{C}$ with growth rate of $300 \mathrm{~nm} / \mathrm{h}$. Although the material is monocrystalline, the surface exhibits a roughness of about $20 \mathrm{~nm}$ and a high value of defects initialized by the low growth temperature instead of the usually used temperatures in the range of $750-900{ }^{\circ} \mathrm{C} .{ }^{11,12}$ The dislocation density is about 5 $\times 10^{9} \mathrm{~cm}^{-2}$ that is 50 times higher than for conventional GaN. MSM structures with finger width and spacing of 1 and $1.5 \mu \mathrm{m}$, respectively, and an active area of $20 \times 20 \mu \mathrm{m}^{2}$, as

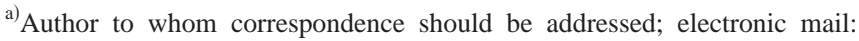
m.mikulics@fz-juelich.de

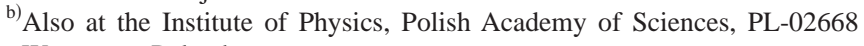
Warszawa, Poland.
}

presented in Ref. 13, were patterned on the GaN surface using conventional photolithography and liftoff technique. The MSM electrodes consist of $\mathrm{Ti} / \mathrm{Al} / \mathrm{Ni} / \mathrm{Au}$ $(35 / 200 / 40 / 50 \mathrm{~nm})$ for forming ohmic contacts. Ohmic contact metallization was annealed at $900{ }^{\circ} \mathrm{C}$ for $30 \mathrm{~s}$. Devices with Schottky contacts $(25 \mathrm{~nm} \mathrm{Ni}, 300 \mathrm{~nm} \mathrm{Au})$ were fabricated for the sake of comparison. The whole surface except the MSM structures was coated with a 400-nm-thick $\mathrm{SiO}_{2}$ layer. Ti/ $\mathrm{Au}$ coplanar strip lines (CPS) with thickness of $50 / 600 \mathrm{~nm}$ were fabricated on top of this insulator layer.

Figure 1 shows typical current-voltage $(I-V)$ characteristics of the photodetectors fabricated on LT-GaN material measured in the dark. The MSM photodetectors with ohmic contacts exhibit linear behavior in the whole bias range up to $100 \mathrm{~V}$. The characteristics of the MSM photodetectors with Schottky contacts is more complex. It is defined by the reverse current of a Schottky contact above GaN with a high defect density. Furthermore the free carriers are collected at a different thickness of the layer structure with different bias voltage. Rapid increase of the dark current at higher bias is due to the collection of carriers in the deeper regions of $\mathrm{GaN}$

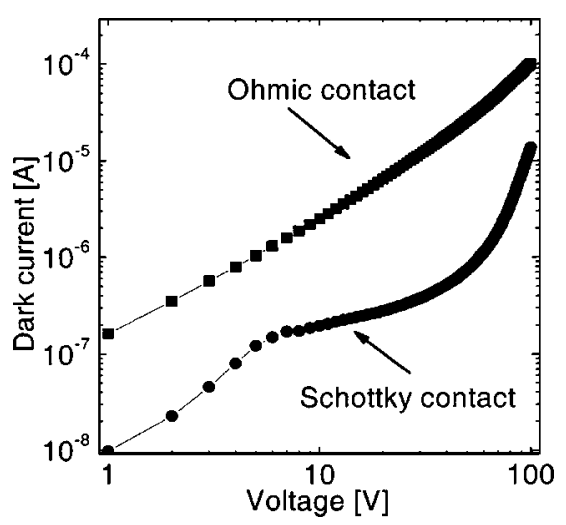

FIG. 1. Current-voltage $(I-V)$ characteristics of the MSM LT GaN photodetectors with ohmic and Schottky contacts, with active area of 20 $\times 20 \mu \mathrm{m}^{2}$ and with finger width and spacing of 1 and $1.5 \mu \mathrm{m}$, respectively. 


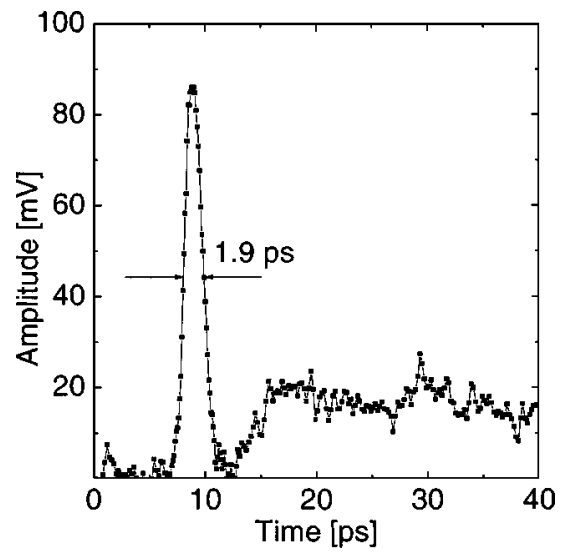

FIG. 2. Time-resolved photoresponse waveform for the MSM LT GaN photodetectors with ohmic contacts (active area: $20 \times 20 \mu \mathrm{m}^{2}$, finger width and spacing: 1 and $1.5 \mu \mathrm{m}$ ), measured at $10 \mathrm{~V}$ bias and $5 \mathrm{~mW}$ input power.

under the AlN layer. The temporally resolved photoresponse of the devices was measured under the illumination with 100-fs-wide, 360-nm-wavelength, 80-MHz-repetition rate optical pulses from a commercial Ti:sapphire laser. The photoresponse waveforms were recorded with the help of an electro-optic (EO) sampling system, featuring 200 fs temporal resolution. ${ }^{14}$ Electrical transients were sampled at a spot on the CPS line located $\sim 30 \mu \mathrm{m}$ away from our device. The time-resolved photoresponse waveform of MSM photodetectors with ohmic contacts measured at $10 \mathrm{~V}$ bias and $5 \mathrm{~mW}$ optical input power is shown in Fig. 2. Photodetector response exhibits full width at half maximum (FWHM) of about $1.9 \mathrm{ps}$ with amplitudes up to $85 \mathrm{mV}$. With the calculated RC time constant of $1.2 \mathrm{ps}$, the carrier lifetime is assumed to be about $1.4 \mathrm{ps}$ at $10 \mathrm{~V}$ bias and $5 \mathrm{~mW}$ optical input power. ${ }^{14}$ To evaluate the measured pulse amplitude: the obtained value for an optimized low temperature grown GaAs photodetector with the same layout and under the same operation conditions (bias voltage: $10 \mathrm{~V}$, optical input power: $5 \mathrm{~mW}$ ) is $700 \mathrm{mV} .{ }^{13}$ Because of the lower wavelength of $360 \mathrm{~nm}$ at the $\mathrm{GaN}$ measurements, compared to $810 \mathrm{~nm}$ for GaAs, the same optical input power gives only $44 \%$ of incident photons for GaN compared to GaAs. Furthermore, the $360 \mathrm{~nm}$ wavelength is only $5 \mathrm{~nm}$ above the band gap of $\mathrm{GaN}^{9}{ }^{9}$ Considering both facts, the sensitivity of the LT-GaN detector is comparable to the devices based on LT-GaAs.

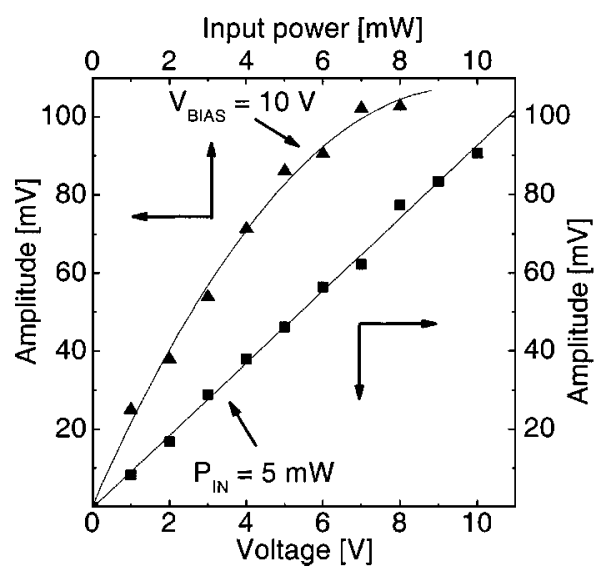

FIG. 3. Photoresponse amplitude of the MSM LT GaN photodetector as a

function of bias voltage and optical input power.
Foxon, J. Cryst. Growth 218, 1 (2000).

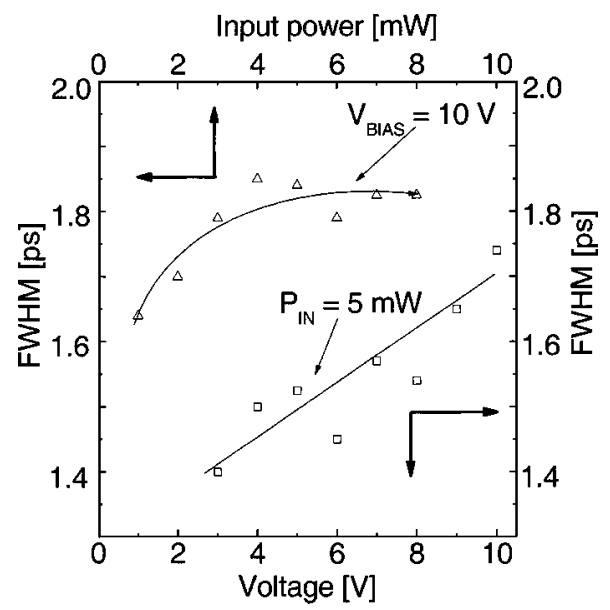

FIG. 4. FWHM of electrical transients of the LT GaN photodetector as a function of bias voltage and input power.

Figure 3 presents the photoresponse amplitude of the photodetector as a function of bias voltage and optical input power. We observe that the photoresponse amplitude increases linearly with increased bias voltage. The dependence on input power is linear for low power and gradually approaches saturation. We attribute this saturation and also the increase of the FWHM with increasing input power observed in Fig. 4 to heating of the device by the optic input power and by the photocurrent. The same behavior is observed when the bias voltage is increased: the FWHM increases for about $25 \%$ with a change of the bias from 3 to $10 \mathrm{~V}$ (Fig. 4). Beside the effect of heating, the increase of the FWHM by increasing the bias can also be caused by an increase of the carrier life time due to a reduction of the electron capture cross section with increased electric field, as it was observed earlier by Zamdmer and $\mathrm{Hu}$ in LT GaAs. ${ }^{15}$ At $3 \mathrm{~V}$ bias and $5 \mathrm{~mW}$ input power, we recorded 1.4 ps-wide-electrical transient that is the shortest measured ultraviolet response for a GaN photodetector reported to date. We must stress, however, that the effective carrier lifetime in our LT GaN materials is extended from $\sim 720$ fs to $\sim 1.4$ ps at high bias and intense input power because of the effects mentioned above. These values are extracted from the experimental results using the method mentioned in Ref. 14.

In conclusion, we have fabricated ultrafast MSM photodetectors on LT GaN grown by plasma induced molecular beam epitaxy. The devices exhibit a peak amplitude up to $100 \mathrm{mV}$ with FWHM as short as $\sim 1.4$ ps photoresponse under illumination by 100 -fs-wide and 360-nm-wavelength laser pulses. These results show that LT GaN material is a promising candidate for high speed UV photodetectors.

${ }^{1}$ M. L. Lee, Y. K. Su, S. J. Chang, W. C. Lai, and G. C. Chi, IEEE Electron Device Lett. 25, 593 (2004).

${ }^{2}$ E. Monroy, E. Munoz, F. J. Sanchez, F. Calle, E. Calleja, B. Beaumont, P. Gibart, J. A. Munoz, and F. Cusso, Semicond. Sci. Technol. 13, 1042 (1998).

${ }^{3}$ G. Y. Xu, A. Salvador, W. Kim, Z. Fan, C. Lu, H. Tang, H. Morkoç, G. Smith, M. Estes, B. Goldenberg, W. Yang, and S. Krishnankutty, Appl. Phys. Lett. 71, 2154 (1997).

${ }^{4}$ E. Monroy, T. Palacios, O. Hainaut, F. Omnès, F. Calle, and J. F. Hochedez, Appl. Phys. Lett. 80, 3198 (2002).

${ }^{5}$ B. Poti, M. T. Todaro, M. C. Frassanito, A. Pomarico, A. Passaseo, M. Lomascolo, R. Cingolani, and M. De Vittorio, Electron. Lett. 39, 1747 (2003).

${ }^{6}$ H. Z. Xu, Z. G. Wang, M. Kawabe, I. Harrison, B. J. Ansell, and C. T.

Foxon, J. Cryst. Growth 218, 1 (2000). 
${ }^{7}$ M. Mosca, J.-L. Reverchon, F. Omnes, and J.-Y. Duboz, J. Appl. Phys. 95, 4367 (2004).

${ }^{8}$ S. Yoshida, J. Cryst. Growth 237, 978 (2002).

${ }^{9}$ J. Li, Y. Xu, T. Y. Hsiang, and R. Donaldson, Appl. Phys. Lett. 84, 2091 (2004).

${ }^{10}$ J. Li, W. R. Donaldson, and T. Y. Hsiang, IEEE Photonics Technol. Lett. 15, 1141 (2003).

${ }^{11}$ J. W. P. Hsu, M. J. Manfra, S. N. G. Chu, C. H. Chen, L. N. Pfeiffer, and
R. J. Molnar, Appl. Phys. Lett. 78, 3980 (2001).

${ }^{12}$ P. Waltereit, S. H. Lim, M. McLaurin, and J. S. Speck, Phys. Status Solidi A 194, 524 (2002).

${ }^{13}$ M. Mikulics, Ph.D. thesis, RWTH Aachen, Germany, 2005.

${ }^{14}$ X. Zheng, Y. Xu, R. Sobolewski, R. Adam, M. Mikulics, M. Siegel, and P. Kordoš, Appl. Opt. 42, 1726 (2003).

${ }^{15}$ N. Zamdmer and Q. Hu, Appl. Phys. Lett. 75, 2313 (1999). 